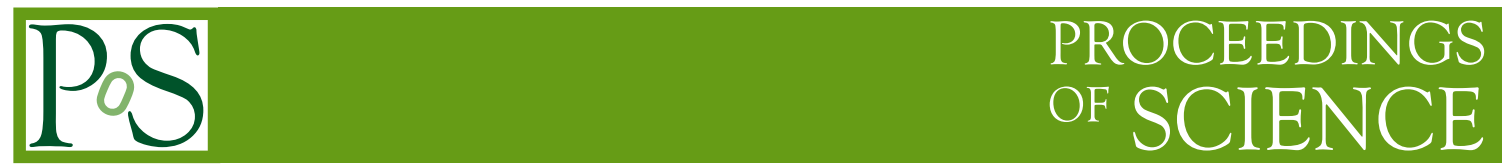

\title{
Searches for invisible Higgs decays at the LHC
}

\section{Andreas Albert ${ }^{a, *}$ for the ATLAS and CMS Collaborations}

${ }^{a}$ Physics Department, Boston University,

590 Commonwealth Avenue, Boston, USA

E-mail: andreas.albert@cern.ch

Searches for decays of the Higgs boson to invisible particles simultaneously probe the standard model nature of the Higgs sector and the possible existence of DM particle candidates. The ATLAS and CMS Collaborations pursue an ambitious search program for such hypothetical decays in various analysis channels. Using the latest data sets from Run-2 of the CERN LHC (corresponding to approximately $140 \mathrm{fb}^{-1}$ per experiment), impressive progress has been made in constraining invisible decays of the Higgs boson. In this talk, I present the latest results, discuss their relative importance and summarize the prospects for the near future.

The Ninth Annual Conference on Large Hadron Collider Physics - LHCP2021

7-12 June 2021

Online

${ }^{*}$ Speaker 
Two of the most prominent fields in particle physics today are the study of the Higgs boson discovered at the LHC [1-3], and the quest for an understanding of the nature of dark matter. These topics intersect in the search for couplings of the Higgs boson to potential dark matter candidates, which would manifest themselves in "invisible" decays of the boson at the LHC. Searches for invisible final states at the LHC rely on missing transverse momentum, or $p_{T}^{\text {miss }}$, which is inferred from a vectorial sum of detected particle momenta and measures of the transverse momentum carried away from a given collision by undetected particles. Searches for invisible decays of the Higgs boson therefore target final state with significant $p_{T}^{\text {miss }}$ accompanied by different sets of standard model "tag" particles, with different tags favoring different signal production modes. In the following, I will present searches performed by the ATLAS and CMS Collaborations [4, 5]. In all cases, SM-like production of the Higgs boson is assumed via the gluon fusion $(\mathrm{ggH})$, vector boson fusion (VBF), $\mathrm{Z}$ or $\mathrm{W}$ associated production $(\mathrm{VH})$, or top quark associated production (ttH) modes. All constraints are quoted at $95 \%$ confidence level.

Searches in the VBF topology are generally leading in sensitivity for invisible Higgs decays, owing to a favorable coincidence of signal cross section and background rejection handles. The final state signature consists of jets and $p_{T}^{\text {miss }}$, with leading standard model (SM) background contributions arising from $\mathrm{Z}(v v)$ and $\mathrm{W}(\ell v)$ production. These contributions can be reduced with requirements on the accompanying jets. The strongest constraints in this channel are set by ATLAS in Ref. [6] based on the full Run-2 data set $\left(139 \mathrm{fb}^{-1}\right)$. In this search, events are selected using a $p_{T}^{\text {miss }}$ trigger and an offline requirement of $p_{T}^{\text {miss }}>200 \mathrm{GeV}$. The two highest- $p_{T}$ jet are required to lie in opposite $\eta$-hemispheres of the detector and to form a pair with high invariant mass, small angular separation and large rapidity gap, which are typical characteristics of VBF production. Events with charged leptons, photons or $\mathrm{b}$ tagged jets are stringently rejected. To further enhance the analysis sensitivity, the signal region (SR) is binned in $m_{j j}, \Delta \phi_{j j}$, and jet multiplicity. A binned maximum likelihood (ML) fit is performed combining the SR with control regions (CRs) where the transverse momentum is replaced by a well-identified $\mathrm{W}$ or $\mathrm{Z}$ candidate with one or two charged leptons. The differential shapes and normalizations of the leading background contributions are thus directly estimated from data, and the resulting background model is shown in Fig. 1. The branching fraction of the Higgs boson to invisible particles is constrained to be less than $13 \%(13 \%$ expected). The most recent corresponding result from CMS uses similar methods based on a dataset of $36 \mathrm{fb}^{-1}$ [7], resulting in an upper limit of $33 \%$ (25\% expected) on the invisible branching fraction $\mathcal{B}_{\text {inv }}$. Beyond the fully hadronic channel, the ATLAS Collaboration has also performed a search for VBF production in association with a photon in the same data set using an orthogonal selection [8]. The properties of the radiated photon, as well as those of the jets, are fed into a neural network, which provides an output score that is used for signal extraction. The main backgrounds from $\mathrm{Z} \gamma$ and $\mathrm{W} \gamma$ production are estimated from simulation, with normalization corrections from control samples in data. This strategy results in an additional constraint of $\mathcal{B}_{\text {inv }}<37 \%$ (34\% expected).

The second-leading channel is $Z(\ell \ell) H$, where $\ell=\mathrm{e}, \mu$. The charged leptons from the $\mathrm{Z}$ decay can be used for triggering, with typical $p_{T}$ thresholds for di-lepton triggers in the $20-30 \mathrm{GeV}$ range. These low trigger thresholds directly translate into lower offline $p_{T}^{\text {miss }}$ thresholds, and thus large signal acceptance. In addition, the characteristic features of the lepton pair are potent handles for effective background rejection. In the currently strongest result in this channel [9], the CMS Collaboration analyzes a data set corresponding to $137 \mathrm{fb}^{-1}$. Events are selected that contain a same- 
flavor lepton pair with $\left|m(\ell \ell)-m_{\mathrm{Z}}\right|<15 \mathrm{GeV}$, as well as $p_{T}^{\text {miss }}>100 \mathrm{GeV}$. Further background rejection is achieved by requiring that the missing momentum and $\mathrm{Z}$ boson are balanced backto-back in the transverse plane $\left(\left|p_{T}^{\text {miss }}-p_{T}(\ell \ell)\right| / p_{T}(\ell \ell)<0.4, \Delta \phi\left(p_{T}^{\text {miss }}, p_{T}(\ell \ell)\right)>2.6\right)$, and subcategorizing SR events based on whether they contain a hadronic jet. The leading backgrounds stem from $\mathrm{ZZ}$ and $\mathrm{ZW}$ production, which are estimated using a ML fit to the SRs and CRs with three or four charged leptons. The resulting constraint is $\mathcal{B}_{i n v}<29 \%$ (25\% expected). In a similar search based on $36 \mathrm{fb}^{-1}$ from the ATLAS Collaboration, $\mathcal{B}_{i n v}$ is constrained to be less than $67 \%$ (39\% expected) [10].

The VH mode can further be pursued in the case of hadronic V decays. The high $p_{T}^{\text {miss }}$ trigger thresholds imply large $\mathrm{V}$ boson momentum, resulting in a characteristic signature where the decay partons are merged into a single wide jet. In a recent search, the CMS Collaboration uses a deep neural network to distinguish such wide jets from jets originating from QCD radiation [11]. Separate analysis categories are defined for events with and without V-like jets, with the latter category being used for the search for the gluon fusion signal. Leading background contributions again arise from $\mathrm{Z}$ and $\mathrm{W}$ production, and are again estimated using a combined ML fit including five CRs (1e, 2e, $1 \mu, 2 \mu, \gamma)$ for each of the SRs. Additional contributions from diboson and top quarks, which are especially relevant in the VH categories, are estimated from simulation. The combined constraint using all regions is $28 \%$ ( $25 \%$ expected). In a similar search focused on ggH-like events in a data set of $139 \mathrm{fb}^{-1}$, the ATLAS Collaboration has recently found that $\mathcal{B}_{\text {inv }}<34 \%$ (39\% expected) [12].

Finally, ttH production differentiates itself from the other modes through typically large final state multiplicities. Analysis approaches developed for supersymmetric partners of the top quark are well suited for this topology. The most stringent bounds in the $\mathrm{ttH}$ mode are set by a reinterpretation of hadronic and leptonic stop searches with the ATLAS experiment, using the full Run-2 data set [13]. The combination of both results in a constraint of $\mathcal{B}_{\text {inv }}<40 \%$ (36\% expected).

Beyond individual channels, the strongest overall constraints are obtained from combinations of searches. The combination procedure is conceptually straightforward, but care must be taken to avoid overlaps between the various regions in different searches, and to properly model uncertainty correlations between them. The best direct constraint has been obtained by the ATLAS Collaboration in a combination of the full Run-2 VBF and ttH results discussed above, as well as additional results from Run- 1 of the LHC [13]. The resulting limit is $11 \%$ (11\% expected). A combination of CMS analyses based on up to $36 \mathrm{fb}^{-1}$ has yielded a result of $\mathcal{B}_{\text {inv }}<19 \%$ (15\% expected) [7]. The results of both combinations are shown in Fig. 2. Beyond combining direct searches, $\mathcal{B}_{\text {inv }}$ can also be seen as a parameter embedded in a broader framework for modified couplings of the Higgs boson. In this case, one can further combine results with searches in visible Higgs boson decay modes ( $\tau \tau, \gamma \gamma, \mathrm{ZZ}$, etc.). Under the assumption that the magnitude of the couplings of the Higgs boson to $\mathrm{Z}$ and $\mathrm{W}$ bosons is smaller or equal to the SM prediction, the constraint from the ATLAS VBF result can be tightened to $9 \%$ [14].

The next milestone in constraining the invisible branching of the Higgs boson is the completion of the channel combinations in both experiments. Based on the results known so far, it is likely that the direct search combinations will reach below the $10 \%$ threshold for $\mathcal{B}_{\text {inv }}$. As some analyses have not yet been completed with full Run-2 data, the extent to which the threshold will be surpassed is the central question. Beyond Run-2, significant gains in sensitivity are expected to come with future data sets at the LHC, as specifically the VBF channel is still statistically limited. 


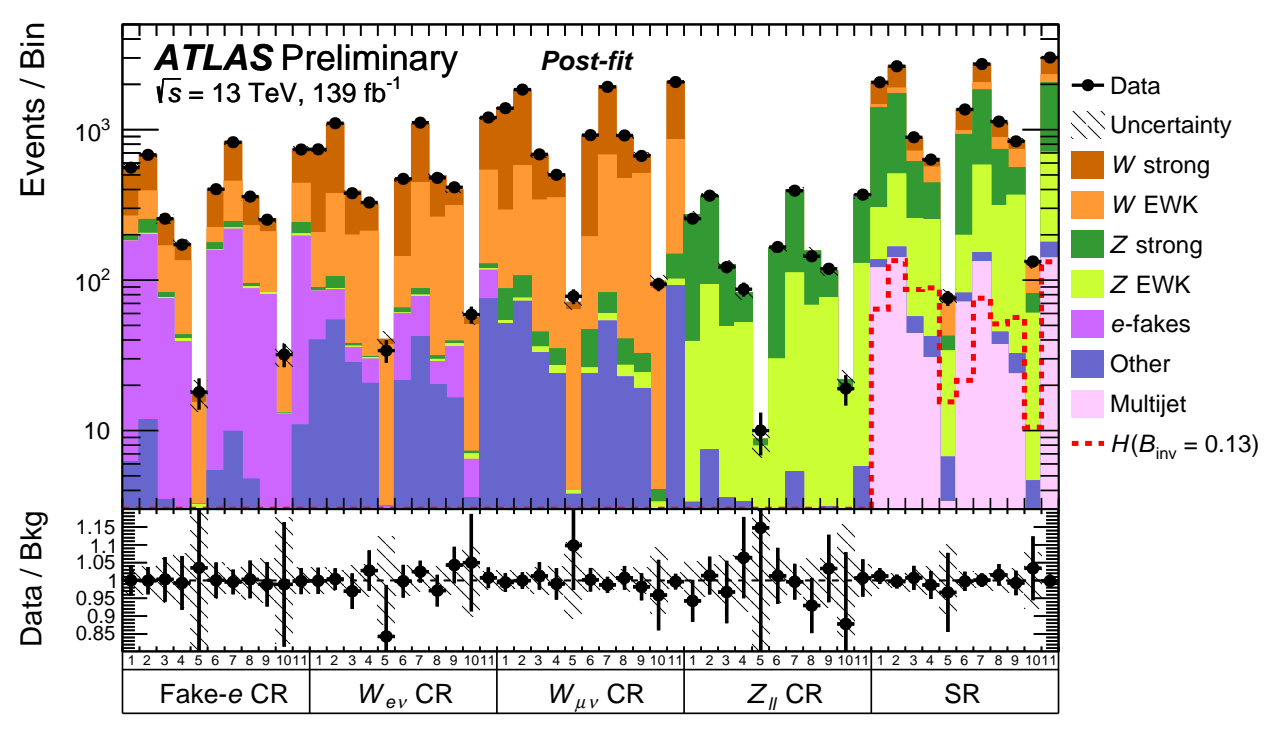

Figure 1: Comparison of data and post-fit background model for the hadronic VBF analysis performed by the ATLAS Collaboration [6]. In each region, the consecutively numbered bins are separated by $m_{j j}$, $\Delta \phi_{j j}$ and jet multiplicity, with the best signal-to-background ratio being achieved in bin $5\left(m_{j j}>5 \mathrm{TeV}\right.$, $\Delta \phi_{j j}<1, N_{j e t}=2$ ). Data (black points) and background estimate (filled histograms) show good agreement. The signal prediction for $\mathcal{B}_{i n v}=13 \%$ is shown as a red dotted line. Taken from Ref. [6].
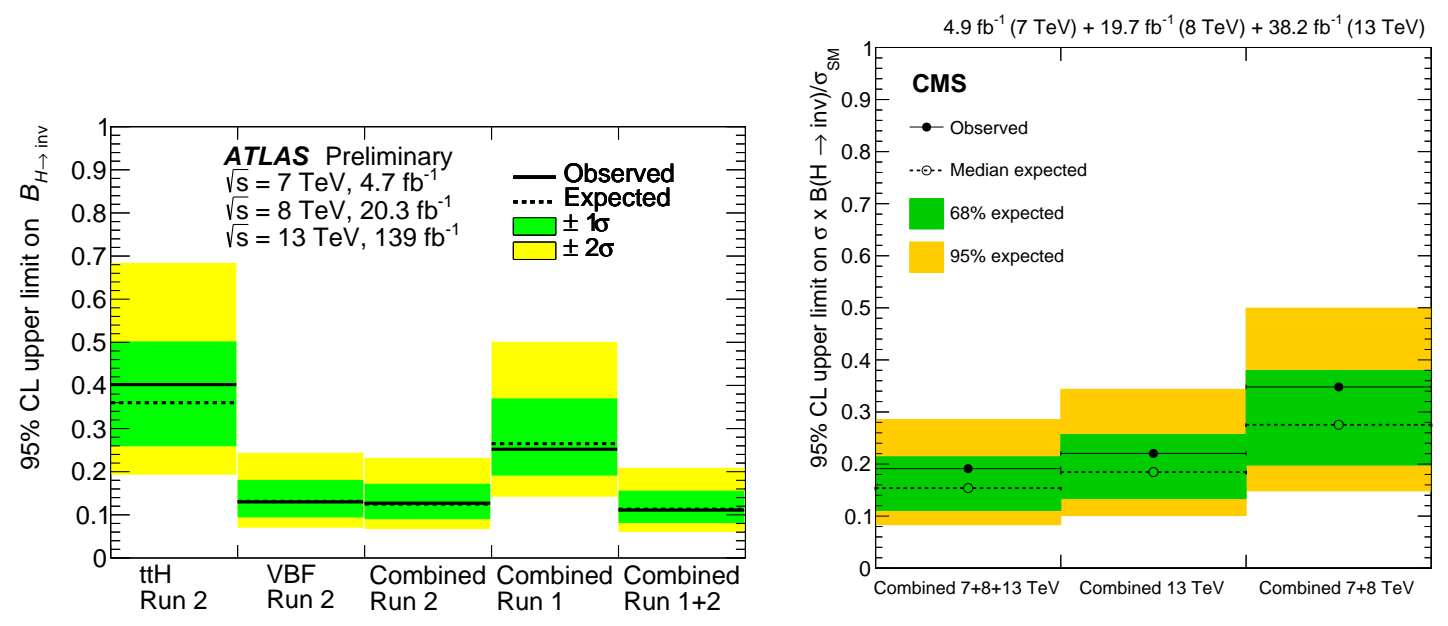

Figure 2: Results of direct search combinations from the ATLAS (left, from Ref. [13]) and CMS Collaborations (right, from [15]). The ATLAS combination includes the full Run-2 searches in the VBF and ttH channels, as well as results from Run-1, resulting in the current best direct search constraint of $\mathcal{B}_{\text {inv }}<11 \%$ (11\% expected). The CMS combination also includes the $\mathrm{VH}$ and $\mathrm{ggH}$ channels, but only uses data sets up to and including the 2016 data-taking, resulting in a constraint of $\mathcal{B}_{\text {inv }}<19 \%$ (15\% expected). 


\section{References}

[1] ATLAS Collaboration, Observation of a new particle in the search for the standard model Higgs boson with the ATLAS detector at the LHC, Phys. Lett. B 716 (2012) 1 [1207. 7214$].$

[2] CMS Collaboration, Observation of a new boson at a mass of $125 \mathrm{GeV}$ with the CMS experiment at the LHC, Phys. Lett. B 716 (2012) 30 [1207 . 7235].

[3] CMS Collaboration, Observation of a new boson with mass near $125 \mathrm{GeV}$ in pp collisions at $\sqrt{s}=7$ and $8 \mathrm{TeV}$, JHEP 06 (2013) 081 [1303.4571].

[4] ATLAS Collaboration, The ATLAS Experiment at the CERN Large Hadron Collider, JINST 3 (2008) S08003.

[5] CMS Collaboration, The CMS Experiment at the CERN LHC, JINST 3 (2008) S08004.

[6] ATLAS Collaboration, Search for invisible Higgs boson decays with vector boson fusion signatures with the ATLAS detector using an integrated luminosity of $139 \mathrm{fb}^{-1}$, ATLAS-CONF-2020-008, CERN, Geneva (2020).

[7] CMS Collaboration, Search for invisible decays of a Higgs boson produced through vector boson fusion in proton-proton collisions at $\sqrt{s}=13 \mathrm{TeV}$, Phys. Lett. B 793 (2019) 520 [1809.05937].

[8] ATLAS Collaboration, Observation of electroweak production of two jets in association with an isolated photon and missing transverse momentum, and search for a Higgs boson decaying into invisible particles at 13 TeV with the ATLAS detector, ATLAS-EXOT-2021-17, Submitted to EPJC [2109.00925].

[9] CMS Collaboration, Search for dark matter produced in association with a leptonically decaying Z boson in proton-proton collisions at $\sqrt{s}=13 \mathrm{TeV}$, Eur. Phys. J. C 81 (2021) 13 [Erratum: Eur. Phys. J. C 81 (2021)] [2008.04735].

[10] ATLAS Collaboration, Search for an invisibly decaying Higgs boson or dark matter candidates produced in association with a $Z$ boson in p collisions at $\sqrt{s}=13$ TeV with the ATLAS detector, Phys. Lett. B 776 (2018) 318 [1708.09624].

[11] CMS Collaboration, Search for new particles in events with energetic jets and large missing transverse momentum in proton-proton collisions at $\sqrt{s}=13 \mathrm{TeV}, \mathrm{CMS}-\mathrm{EXO}-20-004$, Submitted to JHEP [2107. 13021].

[12] ATLAS Collaboration, Search for new phenomena in events with an energetic jet and missing transverse momentum in p collisions at $\sqrt{s}=13 \mathrm{TeV}$ with the ATLAS detector, Phys. Rev. D 103 (2021) 112006 [2102 . 10874].

[13] ATLAS Collaboration, Combination of searches for invisible Higgs boson decays with the ATLAS experiment, ATLAS-CONF-2020-052, CERN, Geneva (2020). 
[14] ATLAS Collaboration, A combination of measurements of Higgs boson production and decay using up to $139 \mathrm{fb}^{-1}$ of proton-proton collision data at $\sqrt{\mathrm{s}}=13 \mathrm{TeV}$ collected with the ATLAS experiment, ATLAS-CONF-2020-027, CERN, Geneva (Aug, 2020).

[15] CMS Collaboration, Search for invisible decays of a Higgs boson produced through vector boson fusion in proton-proton collisions at $\sqrt{s}=13 \mathrm{TeV}$, Phys. Lett. B 793 (2019) 520 [1809.05937]. 